\title{
ACCELERATED DOSING FREQUENCY OF A PULMONARY FORMULATION OF TISSUE PLASMINOGEN ACTIVATOR IS WELL-TOLERATED IN MICE
}

\author{
Kathleen A Stringer, ${ }^{* *}$ Meghan Tobias, ${ }^{*}$ John S Dunn,*Il Jackie Campos, ${ }^{\dagger}$ Zachary Van Rheen,* \\ Mitra Mosharraf ${ }^{\dagger}$ and Rajiv Nayar ${ }^{\dagger}$ \\ *Department of Pharmaceutical Sciences, School of Pharmacy, University of Colorado at Denver and Health Sciences Center, \\ Denver, Colorado, ${ }^{\dagger} H T D$ Biosystems, Hercules, California and ${ }^{\ddagger}$ Department of Clinical, Social and Administrative Sciences, \\ University of Michigan, College of Pharmacy, Ann Arbor, Michigan, USA
}

\section{SUMMARY}

1. Tissue plasminogen activator (tPA) has both fibrinolytic and anti-inflammatory activity. These properties may be useful in treating inflammatory lung diseases, such as acute respiratory distress syndrome (ARDS).

2. We have previously demonstrated the feasibility of targeted pulmonary delivery of tPA. As part of our research to develop a clinically viable pulmonary formulation of tPA, we assessed the tolerability and incidence of haemorrhage associated with the administration of a pulmonary formulation of mouse tPA (pf-mtPA).

3. Intratracheal doses of nebulized pf-mtPA or sterile saline were administered with increasing frequency to male and female B6C3F1 mice. After dosing, the mice entered a recovery period, after which they were killed and their lungs were lavaged and harvested. Post-mortem gross necropsy was performed and all major organs were assessed histologically for haemorrhage. The bronchoalveolar lavage fluid was assessed for markers of lung injury.

4. Mouse tPA that was formulated to mimic a previously characterized human pf-tPA was well tolerated when given intratracheally with increasing dosing frequency. The administration of pf-mtPA did not result in any detectable haemorrhagic-related events or signs of lung injury.

5. The results of the present longitudinal study demonstrate that a maximally feasible dose of pf-mtPA $(3 \mathrm{mg} / \mathrm{kg})$ can be given frequently over a short period of time $(12 \mathrm{~h})$ without haemorrhagic complications. Although these data were generated in a

Correspondence: Kathleen A Stringer, Department of Clinical, Social and Administrative Sciences, University of Michigan, College of Pharmacy, 428 Church Street, Ann Arbor, MI 48109-1065, USA.

Email: stringek@umich.edu

The authors have no industrial links or affiliations that represent or could be perceived as conflicts of interest associated with the work presented in this manuscript. Dr Stringer is not a paid employee of and does not have a financial affiliation with HTD Biosystems, Inc.

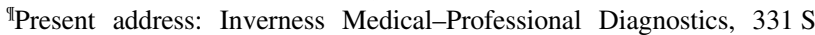
104th St, Louisville, CO 80027, USA.

Received 8 February 2008; revision 23 May 2008; accepted 26 May 2008.

(C) 2008 The Authors

Journal compilation @ 2008 Blackwell Publishing Asia Pty Ltd healthy mouse model, they provide support for the continued evaluation of pf-tPA for the treatment of pulmonary diseases, such as ARDS.

Key words: B6C3F1 mice, haemorrhage, nebulization, pulmonary drug delivery, tissue plasminogen activator.

\section{INTRODUCTION}

Tissue plasminogen activator (tPA) is an endogenous serine protease responsible for fibrin degradation via the activation of plasminogen. Clinically, this function of tPA has been exploited for use in the treatment of myocardial infarction (MI) and thrombotic stroke. More recently, however, mounting evidence suggests that tPA participates in a number of physiological functions that are independent of its fibrinolytic activity. ${ }^{1-4}$ In particular, we have demonstrated the ability of exogenous tPA to suppress activator-induced superoxide anion production by professional phagocytes and to reduce injury in animal models of acute inflammation. ${ }^{5-8}$ Both the fibrinolytic and antiinflammatory properties of tPA could be of use in the treatment of acute respiratory distress syndrome (ARDS), because this illness is characterized by neutrophil-mediated oxidant stress and fibrin deposition in the lungs. ${ }^{9,10}$

We have previously demonstrated the feasibility of a pulmonary formulation of human tPA (pf-tPA). ${ }^{11}$ Targeted pulmonary delivery of tPA would permit direct therapy to the site of drug action and would avoid systemic administration of tPA, which may put patients at undue risk of adverse bleeding events. ${ }^{12,13}$ Haemorrhage is the most serious toxicity of tPA. However, pulmonary delivery of pf-tPA may not be free of bleeding risk, particularly because, unlike in the treatment of MI or stroke, the anticipated clinical situation would require repeated administration. Importantly, to fully assess the tolerability and haemorrhagic potential of pf-tPA, the use of speciesspecific tPA is required because human tPA varies in its ability to activate various animal plasminogen. ${ }^{14}$

Therefore, the purpose of the present study was to test the tolerability of a maximally feasible dose ( $3 \mathrm{mg} / \mathrm{kg}$ ) of mouse pf-tPA (pfmtPA) when given with accelerated frequency to mice. The human pf-tPA formulation parameters established in our previous study were used as benchmarks for the formulation of pf-mtPA. ${ }^{11}$ Several end-points were measured to assess tolerance, the occurrence of haemorrhage and/or lung injury in $\mathrm{B} 6 \mathrm{C} 3 \mathrm{~F} 1$ mice treated with either placebo or nebulized pf-mtPA administered via intratracheal instillation. 


\section{METHODS}

\section{Mouse tPA}

Recombinant mouse tPA (mtPA; Molecular Innovations, Southfield, MI, USA) was formulated to mimic a pulmonary formulation of human recombinant tPA, as described previously. ${ }^{11}$

\section{Assessment of pf-mtPA stability and function}

Protein concentration was assessed by ultraviolet (UV) spectroscopy (HP8453 spectrophotometer; Agilent Technologies, Palo Alto, CA, USA). To determine whether nebulization of pf-mtPA provoked protein self-association, an aggregation index (AI) of pre- and post-nebulized pf-mtPA was determined. ${ }^{11,15}$ The $\mathrm{AI}$ was calculated using the following equation:

$$
\mathrm{AI}=100 \times \mathrm{LS}_{280} /\left(\mathrm{Abs}_{280}-\mathrm{LS}_{280}\right)
$$

where $\mathrm{LS}_{280}$ is the light scattering absorbance (Abs) at $280 \mathrm{~nm}$ determined by linear regression extrapolation through 340,370 and $400 \mathrm{~nm}$. Measurement of self-association is important because the presence of aggregates (e.g. $\mathrm{AI}>10$ ) may contribute to an immune response to the protein. ${ }^{11,16}$

Plasminogen activator inhibitor-1 (PAI-1) is the physiological inhibitor of tPA. ${ }^{17}$ The binding of tPA to PAI-1 is a very specific and high-affinity interaction that results in the complete suppression of the proteolytic activity of tPA, which is responsible for the conversion of plasminogen to plasmin. ${ }^{18,19}$ To test whether the formulation process disrupted the activity of pf-mtPA, pf-mtPA-PAI binding studies were performed using a stable mutant form of human PAI-1 (CPAI) containing four mutations (K154T, Q319L, M354I and $\mathrm{N} 150 \mathrm{H}$; Molecular Innovations). These mutations confer stability to the otherwise labile serpin protein, essentially locking it into its active conformation. Mouse tPA protein $(5 \mu \mathrm{g})$ was incubated in the presence or absence of CPAI $(15 \mu \mathrm{g})$ in Tris-buffered saline (TBS; $\times 1)$ at room temperature for $1 \mathrm{~min}$ to $1 \mathrm{~h}$. Each reaction was stopped by the addition of bioanalyser sample buffer (Agilent Technologies, Santa Clara, CA, USA) and heating to $70^{\circ} \mathrm{C}$ for $10 \mathrm{~min}$. Each denatured sample was then diluted to a final volume of $90 \mu \mathrm{L}$ with water. Samples were assayed and analysed by an Agilent 2100 bioanalyser according to the Protein 200 Plus (Agilent Technologies) assay protocol. This approach uses lab-on-a-chip technology to quantify protein-protein interactions and has been shown to be comparable in sensitivity to sodium dodecyl sulphatepolyacrylamide gel electrophoresis (SDS-PAGE).$^{20}$ Data were analysed using the bioanalyser software.

The fibrinolytic activity of nebulized pf-mtPA was assessed by measuring the cleavage of mouse plasminogen to plasmin. ${ }^{11}$ Each pf-mtPA sample was incubated with mouse plasminogen ( $375 \mu \mathrm{g} / \mathrm{mL}$; Molecular Innovations) for $5 \mathrm{~h}\left(37^{\circ} \mathrm{C}\right)$ in a reaction volume of $50 \mu \mathrm{L}$. Following incubation, the protein was separated by $10 \%$ SDS-PAGE and protein bands were visualized by Coomasie blue.

\section{Nebulization and dose preparation}

The liquid formulation of pf-mtPA was jet nebulized using a Micromist nebulizer (Hudson Respiratory Care, Temecula, CA, USA) with a Sunrise compressor (Model 3655D; Somerset, PA, USA). The collected aerosol was assayed for protein concentration and the AI of pre- and post-nebulized pf-mtPA was determined by UV spectroscopy. ${ }^{11}$ The aerosolization of tPA formulated for pulmonary delivery results in the production of particles with a mass mean diameter and geometric standard deviation of $2.4 \mu \mathrm{m}$ and $2.7 \mu \mathrm{m}$, respectively, which is suitable for delivery to the lower airways. This produces a respirable dose of at least $65 \% .{ }^{11}$ The fibrinolytic activity of pf-mtPA was assessed by measuring the cleavage of plasminogen to plasmin, as described above.

Single dose (approximately $80 \mu \mathrm{L}$ ) aliquots of nebulized pf-mtPA were stored at $4^{\circ} \mathrm{C}$ for no more than 1 week. Before use, the protein concentration and $\mathrm{AI}$ of stored pf-mtPA were measured. Additional pf-mtPA was nebulized as needed. At the time of dosing, the required volume of pf-mtPA or sterile saline was drawn into a sterile syringe $(1 \mathrm{~mL})$ to which a gavage needle $(22 \mathrm{G}$; Hallowell EMC, Pittsfield, MA, USA) was attached. The syringe was prepared so that approximately $100 \mu \mathrm{L}$ air followed the dose.

\section{Animal studies}

The animal protocol was approved by the University of Colorado Health Sciences Center Animal Care and Use Committee and the research adhered to the Principles of Laboratory Animal Care (http://grants.nih.gov/grants/ olaw/references/phspol.htm).

Mice (B6C3F1) were purchased from Taconic Farms (Hudson, NY, USA). Male and female mice (8-9 weeks old) received either a single intratracheal instillation of pf-mtPA ( $3 \mathrm{mg} / \mathrm{kg}$ ) or one dose every $2 \mathrm{~h}$ for 4,8 or $12 \mathrm{~h}$. An equivalent volume of sterile saline (placebo) was given to control mice using the same dosing scheme. Four to six mice were assigned to each group. For dose administration, each mouse was anaesthetized under isoflurane (IsoFlo; isoflurane, USP; Abbott Animal Health, North Chicago, IL, USA), after which the vocal cords were visualized using a rodent intubation table (Hallowell EMC) and a paediatric otoscope (Welch-Allyn Medical Products, Skaneateles Falls, NY, USA). The tip of the gavage needle was gently moved past the vocal cords and into the trachea; the dose of either pf-mtPA or sterile saline was delivered by depression of the syringe's plunger. Following drug or saline administration, the mouse was held upright for approximately $5 \mathrm{~s}$, after which the mouse was returned to the cage and allowed to recover from anaesthesia. Upon the completion of the dosing scheme, animals entered a 2 week recovery period that allowed for the longitudinal assessment of each animal. Because haemorrhage associated with TPA administration is most often acute and symptomatic (e.g. stroke, gastrointestinal), animals were observed twice daily for changes in behaviour and/or function that could be associated with morbidity. ${ }^{12,13,21,22}$ Specifically, lack of grooming, a decline in food and water consumption or urine/faecal production, lethargy or signs of neurological deficit were documented. Respiratory difficulty or distress was considered a study end-point. Nutritional status and well-being were also assessed by changes in weight, for which animals were weighed prior to dosing and on Days 3, 7 and 14. In order to detect haemorrhage in the absence of signs and to account for the possibility of slow, prolonged or asymptomatic bleeding, estimated haematocrit was measured just prior to dosing and at the conclusion of the study. Blood $(50 \mu \mathrm{L})$ was acquired from the retro-orbital sinus by carefully inserting a heparinized capillary tube into the medial canthus at a $30^{\circ}$ angle to the nose while the animal was under isoflurane anaesthesia and a local anaesthetic (proparacaine). Following blood acquisition, pressure was gently applied to the site to ensure the cessation of blood flow before returning the mouse to the cage. Packed red blood cell volume was used as an estimate of haematocrit by subjecting the blood-filled capillary tube to centrifugation and measuring the packed red cell volume as a percentage of the total sample volume.

At the conclusion of the recovery period, mice were killed and their lungs lavaged twice each with $400 \mu \mathrm{L}$ sterile phosphate-buffered saline (PBS). The two broncheoalveolar lavage fluid (BALF) samples were pooled and a cell count was performed using a haemacytometer; a cell differential was performed by fluorescence-activated cell sorting (FACS). The BALF was assayed for protein and lactate dehydrogenase (LDH) activity as indicators of lung injury. ${ }^{23,24}$ Following thoracotomy, the lungs were perfused blood-free with buffered formalin $(10 \%)$ via catheterization of the right ventricle. Subsequently, all animals were perfused blood-free with buffered formalin, after which they were subjected to gross necropsy by a veterinarian blinded to treatment group assignment. Following gross necropsy, the major organs were harvested and paraffin embedded. A single $5 \mu \mathrm{m}$ section from each sample was acquired and stained with haematoxylin and eosin for microscopic histological evaluation. Paraffin embedding, sectioning and staining of organs were performed by the University of Colorado Histology Core Laboratory.

Because the lungs contain endogenous tPA, to characterize the distribution of tPA protein in the lungs following intratracheal administration, mice $(n=3)$ received a single dose $(3 \mathrm{mg} / \mathrm{kg})$ of nebulized human pf-tPA or a matching volume of sterile saline $(n=1)$. Immediately following dose administration, animals were killed and the lungs harvested without lavage. The superior left lobe (which comprises approximately $98 \%$ of the left lung mass) was separated from the inferior left lobe and the three right lobes were separated into the 
superior, mid and inferior lobes. Each lobe from each animal was placed in separate tubes on ice and each was homogenized as described previously. ${ }^{25}$ Each lung lobe homogenate was assayed for human tPA protein concentration by a 96-well microtitre plate enzyme-linked immunosorbent assay. This assay measured total tPA by using a tPA capture antibody (rabbit anti-human tPA; Molecular Innovations) and a different detection antibody (mouse anti-human; CalBiochem, Gibbstown, NJ, USA). The tPA protein was detected following the addition of a secondary antibody (mouse IgG HRP; Millipore, Baillerica, MA, USA) that was quantified by determining the optical density of each well at $450 \mathrm{~nm}$ (SpectraMax Plus; Molecular Devices, Sunnyvale, CA, USA). The tPA concentration in each well was determined from the standard curve by plate reader software. To estimate lung distribution, measured concentrations in each lobe were normalized to $1 \mathrm{~mL}$, from which the amount of protein was determined. The percentage of tPA in each lobe was calculated by dividing this value by total delivered tPA protein and multiplying it by 100 .

\section{Differentiation of BALF cells by FACS}

To determine the percentage of leucocytes (neutrophils, macrophages, lymphocytes) in each BALF sample, cells were pelleted by centrifugation $\left(10000 \mathrm{~g}, 4^{\circ} \mathrm{C}, 5 \mathrm{~min}\right)$ and resuspended in goat serum (2\%) in a Krebs'Ringer-phosphate-dextrose buffer for $1 \mathrm{~h}$. Cells were then pelleted again and resuspended in $100 \mu \mathrm{L}$ staining buffer (1\% bovine serum albumin in PBS) to which the following stains were added: F480 PE (Cal Tag, Burlingame, CA, USA), rat anti-mouse neutrophil-fluorescein isothiocyanate (Cal Tag), antiCD45 PE-Cy7 (BD Bioscience, San Jose, CA, USA), anti-CD3 APC (BD Bioscience), anti-CD45/B220 APC (BD Bioscience) and Live/Dead Fixable Red Dead Cell stain (Invitrogen, Carlsbad, CA, USA). Cells were stained for $20 \mathrm{~min}$ at room temperature. Following staining, cells were washed in staining buffer and fluorescence was detected by a Cytomics FC500 (Beckman Coulter, Fullerton, CA, USA) at the University of Colorado Cancer Center Flow Cytometry Core Laboratory.

\section{Determination of BALF protein concentration and LDH activity}

Protein levels were determined using a micro bicinchoninic acid (BCA) protein assay (Pierce, Rockford, IL, USA). ${ }^{26}$ This assay uses an extended incubation time at an elevated temperature $\left(60^{\circ} \mathrm{C}\right)$, which results in a very sensitive colourimetric protein assay. Leakage of intracellular LDH was measured using an activity assay kit (Sigma-Aldrich, St Louis, MO, USA). Briefly, clarified BALF supernatant $(100 \mu \mathrm{L})$ was added to Reagent A $(2.5 \mathrm{~mL})$ for $1 \mathrm{~min}$, followed by the addition of Reagent B $(100 \mu \mathrm{L})$. The rate of decrease in absorbance $(340 \mathrm{~nm})$ recorded every minute for $3 \mathrm{~min}$ is indicative of the reduction of pyruvate to lactate coupled to the oxidation of NADH to NAD ${ }^{27}$ Absorbance data were analysed using Softmax PRO 4.1 on a ThermoMax microplate reader (Molecular Devices). Lactate dehydrogenase activity data are represented as $\mathrm{NADH}$ consumed $(\mathrm{U} / \mathrm{mL})$.

\section{Data analysis}

Data were analysed by ANOvA (or a two-tailed Student's $t$-test, when applicable) using StatView (SAS, Cary, NC, USA). A Fisher protected least significant difference post hoc analysis was performed where appropriate. In all cases, $P \leq 0.05$ was considered significant.

\section{RESULTS}

The pf-mtPA was proteolytically active, as determined by pf-mtPACPAI binding studies. The protein was primarily single-chain (approximately $85 \%$ ), as evidenced by a $67 \mathrm{kDa}$ single peak at $28 \mathrm{~s}$ (Fig. 1a). Most $(89 \%)$ of the CPAI migrated at $24.5 \mathrm{~s}$ with a molecular weight of $38 \mathrm{kDa}$ (Fig. 1b). The incubation of pf-mtPA with CPAI resulted in the formation of an mtPA-CPAI complex, in which $46 \%$ of the mtPA was bound to CPAI (Fig. 1c). These results are consistent with the manufacturer's characterization of purified mtPA and CPAI (Fig. 1d). Incubation time (1 min to $1 \mathrm{~h}$ ) did not influence mtPA-CPAI complex formation or bioanalyser results and these data were consistent with those observed by SDS-PAGE (Fig. 1d).

In preparation for animal testing, pf-mtPA $(1 \mathrm{mg} / \mathrm{mL})$ was nebulized and protein was assessed for stability and activity. Nebulization did not result in an $\mathrm{AI}>10$, which is consistent with the feasibility criteria established in earlier work (Table 1). ${ }^{11}$ In addition, following nebulization, pf-mtPA retained its fibrinolytic activity (Fig. 2) and the protein concentration and $\mathrm{AI}$ of stored $\left(4^{\circ} \mathrm{C}\right)$ nebulized pf-mtPA remained stable (Table 1).

\section{Animal testing}

All animals survived the dosing and recovery periods. The mean $( \pm$ SEM) age of male and female mice were similar $(9.47 \pm 0.17$ and $9.63 \pm 0.16$ weeks, respectively; $P=0.48$ ), regardless of treatment. There were no detected treatment-related or dosing frequency related haemorrhagic events. There was also no evidence of failure-to-thrive or neurological deficit because there were no apparent changes in animal behaviour or weight.

The administration of both pf-mtPA and placebo was well tolerated. In female mice that received six doses of intratracheal pf-mtPA the mean $( \pm$ SEM) estimated haematocrit decreased from a pretreatment value of $53.5 \pm 0.6 \%$ to a post-treatment value of $46.5 \pm 0.6 \%$ ( $P=0.003$, Student's $t$-test). However, a similar drop in mean haematocrit also occurred in female intratracheal placebo $(\times 6)$-treated mice. Male mice that received either intratracheal pf-mtPA $(\times 6)$ or intratracheal placebo $(\times 6)$ did not have significant pre- or posttreatment changes in mean $( \pm \mathrm{SEM})$ haematocrit (intratracheal pf-mtPA ( $\times 6): 50.0 \pm 0.8 \%$ to $44.5 \pm 3.5 \%(P=0.137)$; intratracheal placebo $(\times 6): 50.5 \pm 0.3 \%$ to $49.7 \pm 0.7 \%(P=0.423))$. However, one male mouse treated with intratracheal pf-mtPA $(\times 6)$ experienced a greater decline in haematocrit (from $50 \%$ to $37 \%$ ) than any of the other mice in the group. This is unexplained because this animal did not have any clinical or pathological evidence of haemorrhage and blood sampling was infrequent, so it is not likely to be the cause. In fact, there was no clinical evidence of haemorrhagic events and conclusive signs of haemorrhage were not identified by gross necropsy in any of the animals. In addition, microscopic assessment of the vitals organs (brain, lungs, heart, liver and kidneys) did not reveal any findings of haemorrhage. Nevertheless, all female mice treated with either six doses of intratracheal pf-mtPA or intratracheal placebo experienced a decline in haematocrit. This decline in haematocrit was only observed in female mice that received six doses of either intratracheal pf-mtPA or intratracheal placebo. In the absence of any signs or pathological evidence of bleeding, this observation may be due to the smaller body mass of the female mice compared with their male counterparts. On average, female mice weighed $20 \%$ less than male mice. The lower bodyweight and corresponding smaller blood volume may have made them more susceptible to a reduction in estimated haematocrit secondary to repeated blood collection despite the use of a conservative blood sampling scheme to attempt to minimize this possibility.

All animals maintained a stable weight during the study period. However, female mice gained less weight over time than male mice. The mean $( \pm$ SEM) weight gain for female intratracheal placebo $(\times 6)$ and intratracheal pf-mtPA $(\times 6)$ mice was $1.2 \pm 0.2$ and $1.6 \pm 0.3 \mathrm{~g}$, respectively, whereas intratracheal placebo $(\times 6)$ and intratracheal pf-mtPA ( $\times 6)$-treated male mice gained $2.5 \pm 0.4$ and $2.3 \pm 0.4 \mathrm{~g}$, 

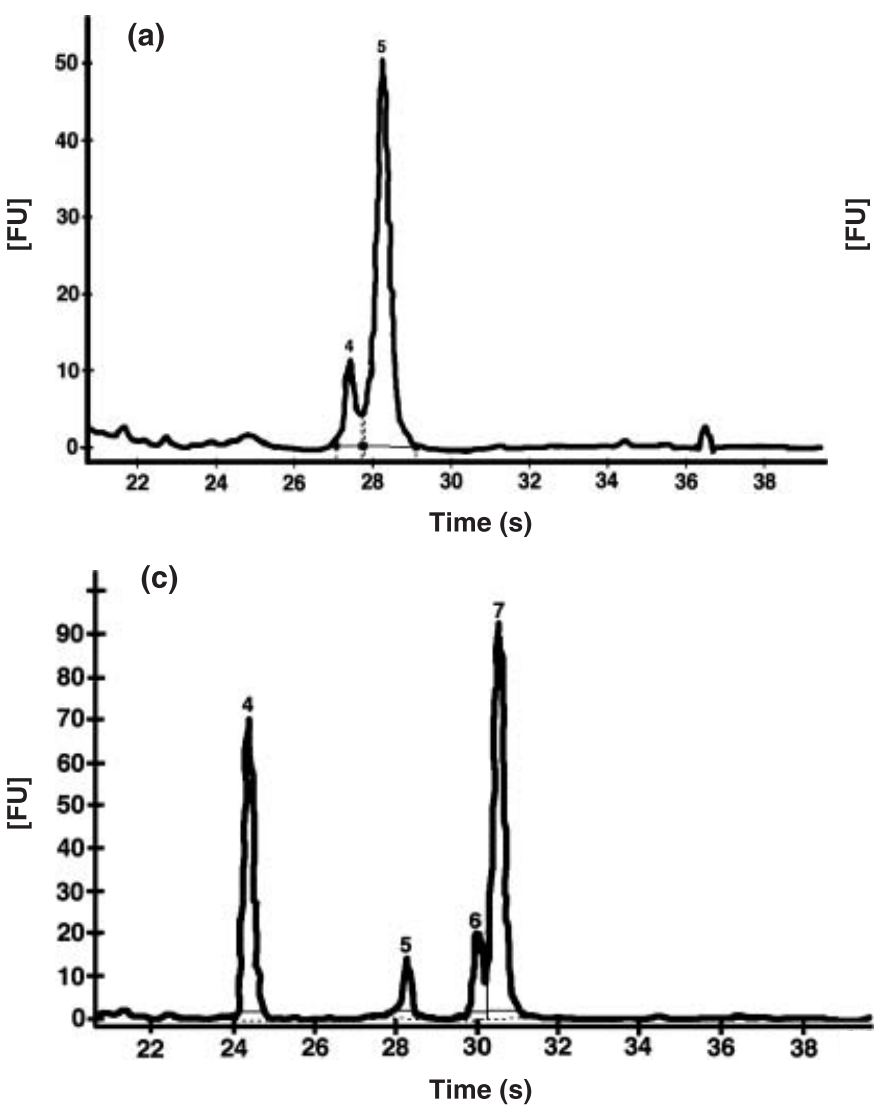

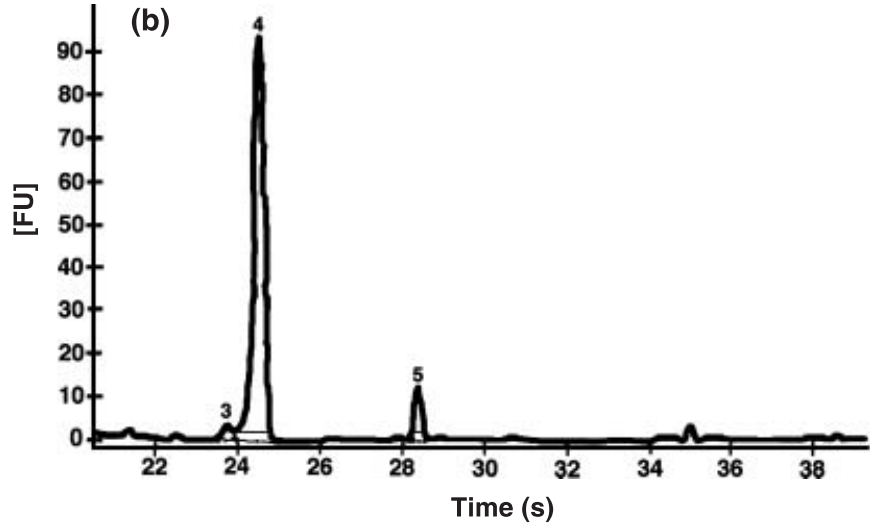

(d)

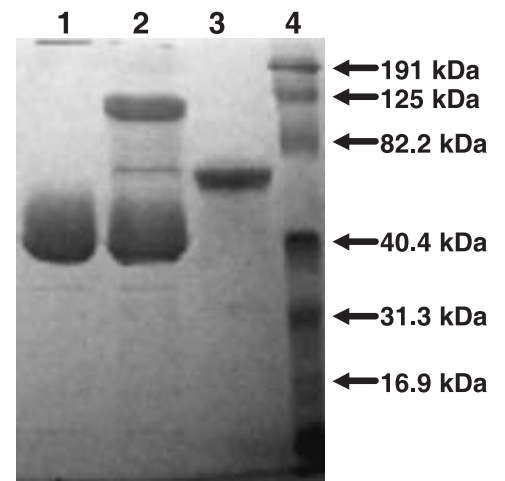

Molecular Innovations, Inc. (Southfield, MI, USA)

Fig. 1 Representative electropherograms of bioanalyser data of a pulmonary formulation of mouse tissue plasminogen activator (pf-mtPA) and interactions between pf-mtPA and a stable mutant form of human plasminogen activator inhibitor-1 (CPAI) at pH 7.3. (a) Analysis of pf-mtPA produced an electropherogram in which most of the protein (85\%) migrated at $28 \mathrm{~s}$ (peak 5). (b) Analysis of CPAI. The two smaller peaks ( 3 and 5) comprise approximately 10\% of the total protein and may be contaminants or by-products. (c) Analysis of pf-mtPA in the presence of CPAI for $1 \mathrm{~h}$ at room temperature produced four peaks (4-7). The mtPA-CPAI complex is represented by peak 7 at 30-31 s (98 kDa). (d) Activity and purity of mtPA by $10 \%$ sodium dodecyl sulphate-polyacrylamide gel electrophoresis following GelCode Stain. Lane 1, CPAI (15 $\mu \mathrm{g})$; lane 2, CPAI $(15 \mu \mathrm{g})$ and $\mathrm{mtPA}(5 \mu \mathrm{g})$ resulted in a bound CPAI-mtPA complex ( 100 kDa); lane 3, mtPA (5 $\mu \mathrm{g})$; lane 4, molecular weight marker (figure provided by Molecular Innovations).

Table 1 Storage $\left(\right.$ at $\left.4^{\circ} \mathrm{C}\right)$ did not alter the protein concentration or aggregation index of the pulmonary formulation of mouse tissue plasminogen activator (pf-mtPA)

\begin{tabular}{lcc}
\hline Condition & pf-mtPA $(\mathrm{mg} / \mathrm{mL})$ & Aggregation index \\
\hline Prenebulized & $1.10 \pm 0.00$ & $1.28 \pm 0.04$ \\
Freshly nebulized & $1.21 \pm 0.01$ & $1.58 \pm 0.34$ \\
Post-nebulization (48 h) & $1.19 \pm 0.00$ & $2.89 \pm 1.50$ \\
Post-nebulization (1 week) & $1.21^{\dagger}$ & $1.56^{\dagger}$ \\
\hline
\end{tabular}

Data are the mean \pm SEM from at least three samples, except for those indicated by the dagger $\left({ }^{\dagger}\right)$, which are for a single sample.

Fig. 2 Nebulization and storage did not alter fibrinolytic activity of a pulmonary formulation of mouse tissue plasminogen activator (pf-mtPA). Representative sodium dodecyl sulphate-polyacrylamide gel electrophoresis (SDS-PAGE) gel (10\%) showing fibrinolytic activity of pf-mtPA (lane $1,50 \mu \mathrm{g} / \mathrm{mL}$; lane 2, $100 \mu \mathrm{g} / \mathrm{mL}$; lane 3, $250 \mu \mathrm{g} / \mathrm{mL}$ ) just after nebulization (a) or after 1 week of storage at $4^{\circ} \mathrm{C}(\mathrm{b})$ following incubation with mouse plasminogen $(375 \mu \mathrm{g} / \mathrm{mL})$ for $5 \mathrm{~h}\left(37^{\circ} \mathrm{C}\right)$. Samples were subjected to SDS-PAGE along with mouse plasminogen and plasmin and molecular weight markers (MWM) for protein identification and size determination. Protein band density is less in (b) than in (a) because less protein was loaded.
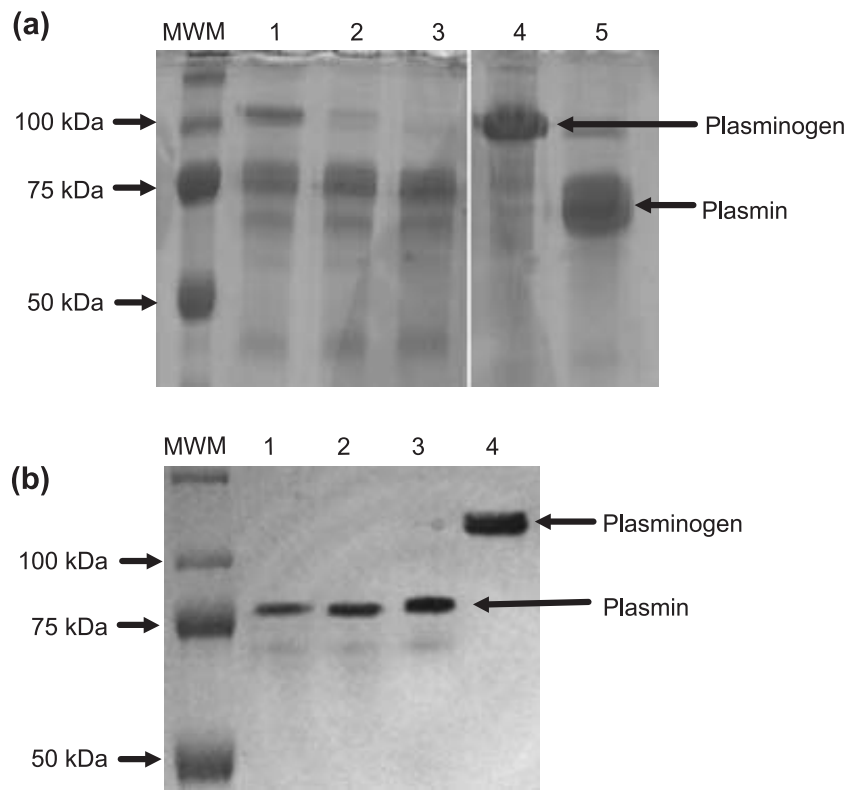


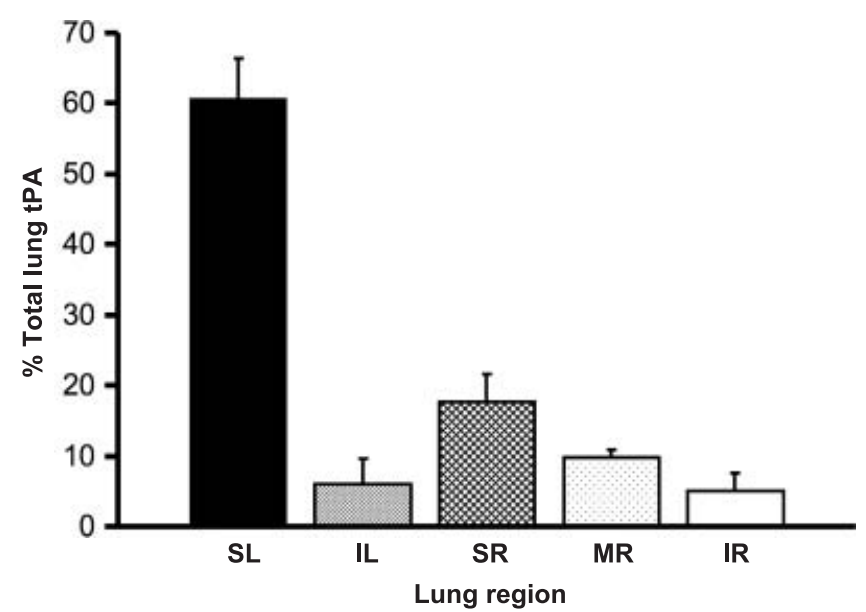

Fig. 3 Distribution of tissue plasminogen activator protein in the lobes of mouse lungs following a single intratracheal dose $(3 \mathrm{mg} / \mathrm{kg})$ of a nebulized pulmonary formulation of human tissue plasminogen activator (pf-tPA). SL, superior left lobe; IL, inferior left lobe; SR, superior right lobe; MR, mid-right lobe; IR, inferior right lobe. Data are the mean \pm SEM from three mice.

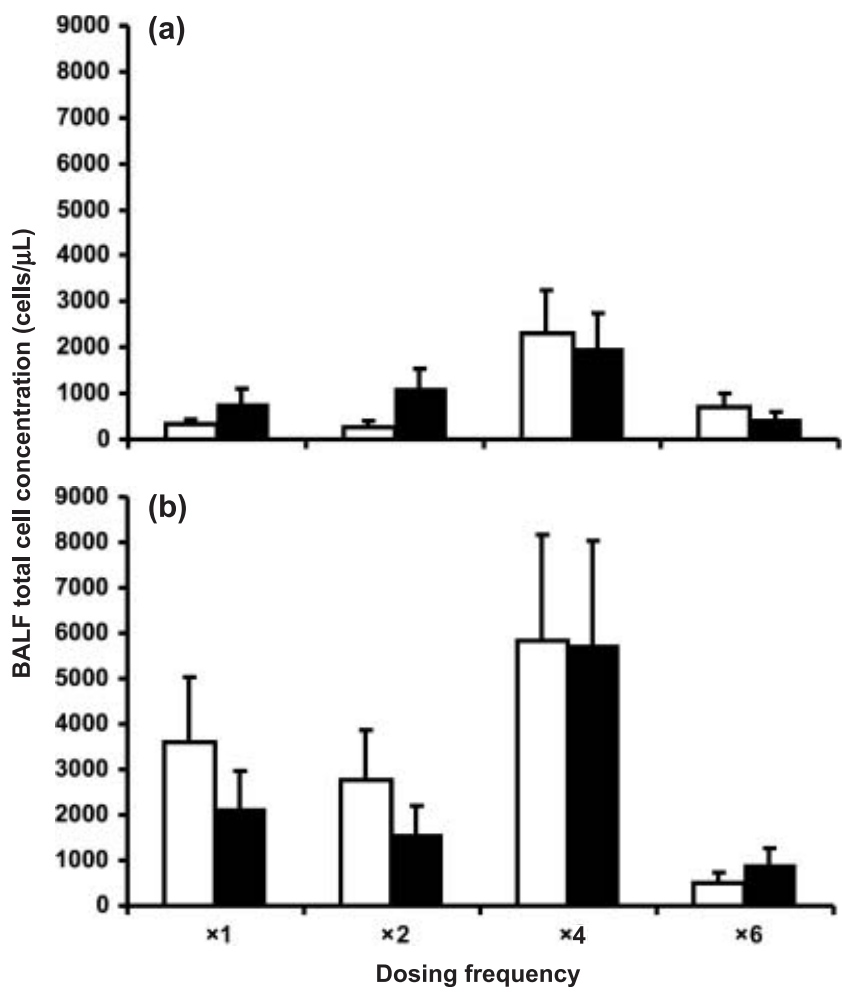

Fig. 4 Increasing dosing frequency of (a) female and (b) male mice with either intratracheally administered placebo $(\square)$ or a pulmonary formulation of mouse tissue plasminogen activator (pf-mtPA; $\mathbf{\square}$ ) was not associated with changes in broncheoalveolar lavage (BALF) cell concentration or inflammatory cell differential. Female mice (a) had consistently lower BALF cell concentrations than male mice (b), regardless of treatment (pf-mtPA $v s$ placebo) or dosing frequency $(P<0.0001)$. There were no differences between pf-mtPAand placebo-treated male and female mice and most cells in all groups were macrophages (see text). Data are the mean \pm SEM of four to six mice per group.

respectively. This is most likely related to sex because age-related differences did not account for this observation.

Tissue plasminogen activator protein was detected throughout the lungs following a single intratracheal dose of nebulized human pf-tPA

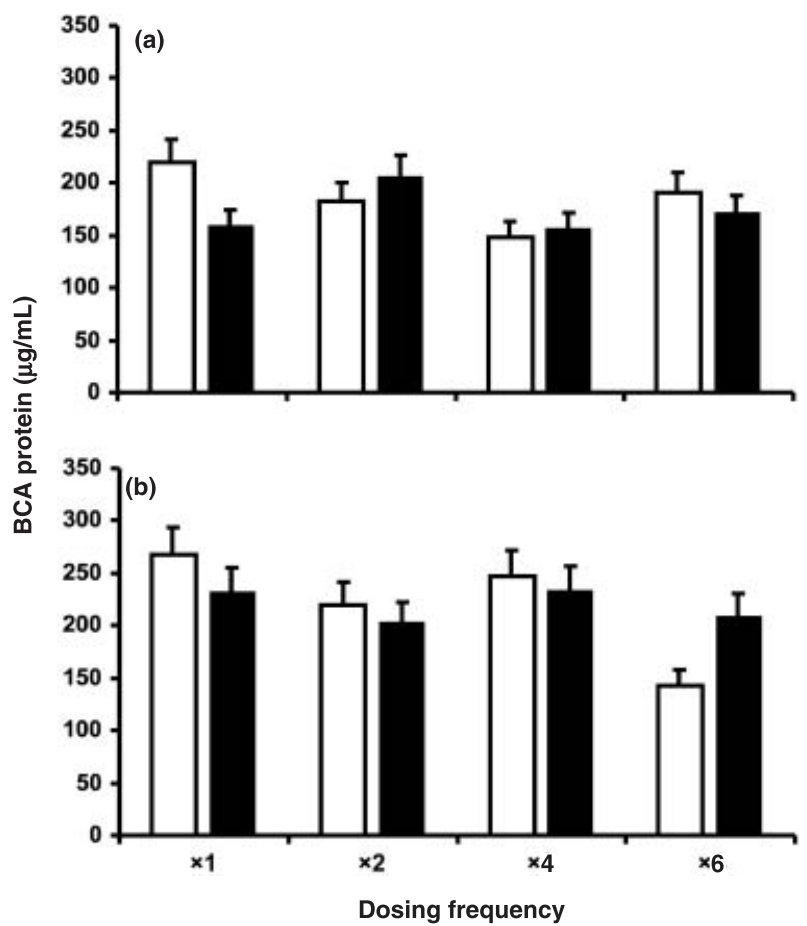

Fig. 5 Increasing dosing frequency of (a) female and (b) male mice with either intratracheal placebo $(\square)$ or a pulmonary formulation of mouse tissue plasminogen activator (pf-mtPA; ) was not associated with changes in broncheoalveolar lavage fluid (BALF) protein concentration, as determined by the BCA assay. Female mice (a) had lower BALF protein concentrations compared with male mice (b) regardless of treatment (pf-mtPA $v s$ placebo) or dosing frequency $(P=0.0114)$. These results are consistent with those shown in Fig. 4 because male mice have a more pronounced pulmonary response to intratracheal administration (see text). Data are the mean \pm SEM of four to six mice per group.

(Fig. 3). Approximately $20 \%$ of the delivered protein reached the lower lobes of the lungs. There was no detectable human tPA in the plasma samples collected. Given the diffuse and distal distribution of tPA, it is reasonable to assume that, consistent with our previous data, some particles reached the alveoli whereas some remained in the larger airways. Regardless, there was no evidence of haemorrhage in either the parenchyma or the larger airway tissues by histology.

Neither pf-mtPA treatment nor increasing dosing frequency resulted in lung inflammation or injury compared with intratracheal placebo, as evidenced by a lack of difference in BALF cell counts (Fig. 4), protein (Fig. 5) and LDH activity between pf-mtPA- and placebo-treated mice. These results demonstrate that intratracheal administration of pf-mtPA given up to six times over a $12 \mathrm{~h}$ period was well tolerated by the lungs. However, regardless of treatment and dosing frequency, the mean BALF cell concentration was higher in male mice than in female mice (Fig. 4). This is most likely sex related because sex differences in mouse pulmonary function and response have been described previously. ${ }^{28}$ Specifically, male mice have been shown to have a more profound inflammatory response to oropharyngeal aspiration of lipopolysaccharide (LPS), as evidenced by higher BALF inflammatory cell counts. ${ }^{28}$ Furthermore, there is also strain variation in both pulmonary function and pulmonary inflammatory responses. ${ }^{29,30}$ Notably, in the present study, the intratracheal administration of pf-mtPA was not associated with an inflammatory response regardless of dosing frequency. Comparatively, untreated male mice 
$(n=4)$ that were not subjected to intratracheal instrumentation had a mean $\left( \pm\right.$ SEM) BALF cell concentration of $18.4 \pm 3.0 \times 10^{4}$ cells $/ \mathrm{mL}$ that was not different from any BALF concentration other than the placebo and tPA $(\times 4)$ male mice $(P=0.005$; Fig. $4 \mathrm{~b})$. In addition, these cells were mostly macrophages $(82.8 \pm 2.7 \%)$. Most of the cells $(\geq 80 \%)$ in the BALF in male and female mice treated with either IPA or placebo were macrophages, regardless of dosing frequency (data not shown). These data suggest that there is no reason to conclude that intratracheal administration of pf-mtPA results in an inflammatory response in the lungs.

The sex difference was also apparent in measured BALF BCA protein concentration. Male mice, regardless of treatment or dosing frequency, had higher BALF protein concentration compared with female mice (Fig. 5). However, LDH activity was below the level of detection $(<0.04 \mathrm{U} / \mathrm{mL})$ in all samples, regardless of sex, treatment or dosing frequency (data not shown), providing evidence that intratracheal administration of pf-tPA was not associated with lung injury.

Collectively, the BALF data support the concept that intratracheal administration of pf-mtPA was well tolerated and was not associated with an increase in measured markers of lung inflammation or injury.

\section{DISCUSSION}

The present study demonstrated the tolerability of a pulmonary formulation of mouse tPA when given with increasing frequency. The significance of this study is that it provides evidence for the safety of the pulmonary route of delivery for a new and novel formulation of tPA. The use of a species-specific therapeutic protein formulation is particularly important for the accurate assessment of tPA toxicity because there is species variation in the tPA substrate plasminogen. ${ }^{14}$ In addition, species-specific therapeutic protein formulations that imitate the human product could assist in addressing the growing need for animal models of drug-disease interactions that can assist in predicting successful translation of preclinical data to safety and efficacy in the clinical situation.

In the mouse model used in the present study, we tested a maximally feasible dose $(3 \mathrm{mg} / \mathrm{kg})$ of pf-mtPA, given with ascending dosing frequency. The maximally feasible dose was determined based on the amount (volume) of drug that could reasonably be administered as a single dose (approximately $80 \mu \mathrm{L}$ ). This approach was used in order to characterize the 'worse case' clinical scenario, which would be the frequent administration of a higher than expected dose. Despite an aggressive dosing frequency of every $2 \mathrm{~h}$ and the administration of up to six doses over a period of $12 \mathrm{~h}$, none of the animals experienced an acute haemorrhagic event (e.g. stroke) or respiratory complications during the dosing period. There were also no apparent late systemic or pulmonary haemorrhagic consequences, as evidenced by a lack of change in estimated haematocrit during the recovery phase and the results of gross necropsy and histological assessment of target organs. However, we acknowledge that our ability to assess asymptomatic bleeding during the recovery may have been limited by a conservative blood sampling scheme $(\leq 100 \mu \mathrm{L}$ over 14 days), which we used to try to reduce the likelihood of anaemia secondary to blood sampling. Despite this, if physiologically relevant bleeding occurred, we would expect it to have been detected by either gross necropsy or histology, which it was not. Furthermore, none of the animals demonstrated signs consistent with bleeding, such as respiratory distress, neurological deficit or failure-to-thrive, changes in grooming, a decline in food and water consumption or urine/faecal production, lethargy or weight loss.
The purpose of developing a pulmonary formulation of tPA is to circumvent the systemic circulation and allow for targeted pulmonary delivery of tPA to the site of drug action. Our previous work has demonstrated that nebulization of a pulmonary formulation of tPA produced particles that can feasibly reach the lower airways. ${ }^{11}$ Given our objective to target treatment to the lungs, it would be preferably that lung-delivered tPA not reach the systemic circulation. Importantly, for protein therapeutics, particle size alone does not predict systemic delivery; there are a number of other variables that contribute. ${ }^{31}$ These include protein size, hydrophobicity and cellular uptake or tissue binding. Furthermore, route of administration (in the case of the present study, intratracheal administration of nebulized protein, which involves pushing drug into the airways using a syringe) will also influence particle distribution in the lungs. Nevertheless, this approach did result in diffuse distribution of the protein in the lungs, with approximately $20 \%$ of protein reaching the lower lungs.

Although the present study was not designed to evaluate the systemic absorption of pulmonary delivered tPA, tPA was not detected in plasma samples collected following a single dose of nebulized human pf-tPA. Thus, our results suggest that pulmonary administration of pf-mtPA, an approximately $68 \mathrm{kDa}$ protein, did not cause a sufficient increase in systemic mtPA concentration to provoke bleeding. It is important to recognize that because we did not measure systemic mtPA concentrations or fibrinolytic activity in the blood following multiple doses or over time, it is possible that both increased without the occurrence of bleeding. Previous work by others in pigs showed that, following traumatic injury, the single administration of nebulized recombinant single chain urokinase (UK; approximately $5.7 \mathrm{mg} / \mathrm{kg}$ ) was associated with a transient but small increase in systemic UK concentration but did not result in a detectable increase in systemic fibrinolytic activity. ${ }^{32}$ Because the purpose of our study was to test the tolerability of pf-mtPA, a separate study is underway to determine the pharmacokinetics of lung-delivered pf-tPA, in which a comprehensive assessment of the pulmonary to plasma distribution of pf-tPA will be made.

We chose to administer nebulized pf-mtPA intratracheally. Unfortunately, there is no optimal or ideal model of experimental pulmonary drug delivery. The advantages of intratracheal administration are conservation of material and more precise dosing because the drug is delivered directly into the airway. Alternatively, aersolization of drug into a chamber results in inconsistent delivery of drug into the lungs owing to variability in breathing patterns and the proximity of animals to the aerosol emission site. Furthermore, mice are nose breathers, so chamber inhalation results in a significant amount of drug deposition into their nasal passages, as well as significant animal-to-animal variability in the amount of drug that enters the deep lungs. Our work and that by others has demonstrated that intratracheal administration results in efficient delivery of liquid to the distal lungs. ${ }^{33}$ Therefore, these data, coupled with previous data that characterized pf-tPA airway distribution, are representative of the anticipated clinical results following the nebulization of human pf-tPA into normal human lungs.

We conducted our experiments in $\mathrm{B} 6 \mathrm{C} 3 \mathrm{~F} 1$ mice. This is a first generation $\left(\mathrm{F}_{1}\right)$ hybrid strain produced by crossing C57BL/6 females with $\mathrm{C} 3 \mathrm{H}$ males. The advantage of an $\mathrm{F}_{1}$ hybrid is genetic and phenotypic uniformity that minimizes animal-to-animal variability. In addition, this strain is frequently used for a broad range of toxicological and pharmacological studies. ${ }^{34-36}$ These were, however, healthy mice with normal lungs. In the expected clinical situation of ARDS, for example, the lungs would be inflamed with an associated increase in vascular 
permeability. Therefore, no conclusions can be made about the potential haemorrhagic risk that may be present following pulmonary administration of pf-tPA in diseased lungs. Planned future work is directed at assessing efficacy and toxicological end-points in a mouse model of lung inflammation.

In conclusion, the results of the present study demonstrate that a high dose of a pulmonary formulation of mtPA that mimics the human pulmonary formulation of tPA can be given with increasing frequency without causing systemic or lung haemorrhage or lung injury. Although these data were generated in a healthy mouse model, they provide support for the continued evaluation of the usefulness of pf-tPA for the treatment of pulmonary diseases, such as ARDS.

\section{ACKNOWLEDGEMENTS}

This work was supported by grant no. HL071439 from the National Heart, Lung and Blood Institute. The content is solely the responsibility of the authors and does not necessarily represent the official views of the National Heart, Lung and Blood Institute or the National Institutes of Health. The authors acknowledge Karen Helm (University of Colorado Cancer Center Flow Cytometry Core) for her invaluable assistance.

\section{REFERENCES}

1. Cooper P, Potter S, Mueck B, Yousefi S, Jarai G. Identification of genes induced by inflammatory cytokines in airway epithelium. Am. J. Physiol. Lung Cell Mol. Physiol. 2001; 280: L841-52.

2. Stringer KA, Dunn JS, Gustafson DL. Administration of exogenous tissue plasminogen activator reduces oedema in mice lacking the tissue plasminogen activator gene. Clin. Exp. Pharmacol. Physiol. 2004; 31: 327-30.

3. Hu K, Yang J, Tanaka S, Gonias SL, Mars WM, Liu Y. Tissue-type plasminogen activator acts as a cytokine that triggers intracellular signal transduction and induces matrix metalloproteinase-9 gene expression. $J$. Biol. Chem. 2006; 281: 2120-7.

4. Renckens R, Roelofs JJ, Florquin S et al. Endogenous tissue-type plasminogen activator is protective during Escherichia coli-induced abdominal sepsis in mice. J. Immunol. 2006; 177: 1189-96.

5. Stringer KA, Bose SK, McCord JM. Antiinflammatory activity of tissue plasminogen activator in the carrageenan rat footpad model. Free Radic. Biol. Med. 1997; 22: 985-8.

6. Stringer KA. Tissue plasminogen activator inhibits reactive oxygen species production by macrophages. Pharmacotherapy 2000; 20: 375-9.

7. Stringer KA, Lindenfeld J, Repine AJ, Cohen Z, Repine JE. Tissue plasminogen activator (tPA) inhibits human neutrophil superoxide anion production in vitro. Inflammation 1997; 21: 27-34.

8. Stringer KA, Hybertson BM, Cho OJ, Cohen Z, Repine JE. Tissue plasminogen activator (tPA) inhibits interleukin-1 induced acute lung leak. Free Radic. Biol. Med. 1998; 25: 184-8.

9. MacLaren R, Stringer KA. Emerging role of anticoagulants and fibrinolytics in the treatment of acute respiratory distress syndrome. Pharmacotherapy 2007; 27: 860-73.

10. Matthay MA, Zimmerman GA. Acute lung injury and the acute respiratory distress syndrome: Four decades of inquiry into pathogenesis and rational management. Am. J. Respir. Cell Mol Biol. 2005; 33: 319-27.

11. Dunn JS, Nayar R, Campos J et al. Feasibility of tissue plasminogen activator formulated for pulmonary delivery. Pharm. Res. 2005; 22: 1700-7.

12. Gardell SJ. The search for the ideal thrombolytic agent: Maximize the benefit and minimize the risk. Toxicol. Pathol. 1993; 21: 190-8.

13. Simoons ML. Risk-benefit of thrombolysis. Cardiol. Clin. 1995; 13: $339-45$.

14. Korninger C, Collen D. Studies on the specific fibrinolytic effect of human extrinsic (tissue-type) plasminogen activator in human blood and in various animal species in vitro. Thromb. Haemost. 1981; 46: 561-5.

15. Kueltzo L. Ultraviolet absorption spectroscopy. In: Jiskoot W, Crommelin DJ (eds). Methods for Structural Analysis of Protein Pharmaceuticals. American Association of Pharmaceutical Scientists, Arlington. $2005 ; 1-25$.

16. Hermeling S, Schellekens H, Maas C, Gebbink MF, Crommelin DJ, Jiskoot W. Antibody response to aggregated human interferon alpha2b in wild-type and transgenic immune tolerant mice depends on type and level of aggregation. J. Pharm. Sci. 2006; 95: 1084-96.

17. Sherman PM, Lawrence DA, Verhamme IM, Paielli D, Shore JD, Ginsburg D. Identification of tissue-type plasminogen activator-specific plasminogen activator inhibitor-1 mutants. Evidence that second sites of interaction contribute to target specificity. J. Biol. Chem. 1995; 270: 9301-6.

18. MacDonald ME, van Zonneveld AJ, Pannekoek H. Functional analysis of the human tissue-type plasminogen activator protein: The light chain. Gene 1986; 42: 59-67.

19. van Zonneveld AJ, Veerman H, MacDonald ME, van Mourik JA, Pannekoek H. Structure and function of human tissue-type plasminogen activator (t-PA). J. Cell. Biochem. 1986; 32: 169-78.

20. Kuschel M, Neumann T, Barthmaier P, Kratzmeier M. Use of lab-ona-chip technology for protein sizing and quantitation. J. Biol. Tech. 2002; 13: $172-8$.

21. Lauer JE, Heger JJ, Mirro MJ. Hemorrhagic complications of thrombolytic therapy. Chest 1995; 108: 1520-3.

22. Lansberg MG, Albers GW, Wijman CA. Symptomatic intracerebral hemorrhage following thrombolytic therapy for acute ischemic stroke: A review of the risk factors. Cerebrovasc. Dis. 2007; 24: 1-10.

23. Kristof AS, Goldberg P, Laubach V, Hussain SNA. Role of inducible nitric oxide synthase in endotoxin-induced acute lung injury. Am. $J$. Respir. Cell Mol. Biol. 1998; 158: 1883-9.

24. Parker JC, Townsley MI. Evaluation of lung injury in rats and mice. Am. J. Physiol. Lung Cell Mol. Physiol. 2004; 286: L231-46.

25. Stringer KA, Freed BM, Dunn JS, Sayers S, Gustafson DL, Flores SC. Particulate phase cigarette smoke increases MnSOD, NQO1, and CINC-1 in rat lungs. Free Radic. Biol. Med. 2004; 37: 1527-33.

26. Smith PK, Krohn RI, Hermanson GT et al. Measurement of protein using bicinchoninic acid. Anal. Biochem. 1985; 150: 76-85.

27. Decker T, Lohmann-Matthes ML. A quick and simple method for the quantitation of lactate dehydrogenase release in measurements of cellular cytotoxicity and tumor necrosis factor (TNF) activity. J. Immunol. Methods 1988; 115: 61-9.

28. Card JW, Carey MA, Bradbury JA et al. Gender differences in murine airway responsiveness and lipopolysaccharide-induced inflammation. $J$. Immunol. 2006; 177: 621-30.

29. Reinhard C, Eder G, Fuchs H, Ziesenis A, Heyder J, Schulz H. Inbred strain variation in lung function. Mamm. Genome 2002; 13: 429-37.

30. Li YJ, Kawada T, Matsumoto A et al. Airway inflammatory responses to oxidative stress induced by low-dose diesel exhaust particle exposure differ between mouse strains. Exp. Lung Res. 2007; 33: 227-44.

31. Patton JS, Byron PR. Inhaling medicines: Delivering drugs to the body through the lungs. Nat. Rev. Drug Discov. 2007; 6: 67-74.

32. Munster AM, Rasmussen L, Sidelmann J, Ingemann Jensen J, Bech B, Gram J. Effects of inhaled plasminogen activator on the balance between coagulation and fibrinolysis in traumatized pigs. Blood Coagul. Fibrinolysis 2002; 13: 591-601.

33. Su X, Looney M, Robriquet L, Fang X, Matthay MA. Direct visual instillation as a method for efficient delivery of fluid into the distal airspaces of anesthetized mice. Exp. Lung Res. 2004; 30: 479-93.

34. Lynch DW, Placke ME, Persing RL, Ryan MJ. Thirteen-week inhalation toxicity of $\mathrm{N}, \mathrm{N}$-dimethylformamide in $\mathrm{F} 344 / \mathrm{N}$ rats and $\mathrm{B} 6 \mathrm{C} 3 \mathrm{~F} 1$ mice. Toxicol. Sci. 2003; 72: 347-58.

35. Irwin RD. A review of evidence leading to the prediction that 1,4-butanediol is not a carcinogen. J. Appl. Toxicol. 2006; 26: 72-80.

36. Dill J, Fuciarelli A, Lee K, Mellinger K, Burka L, Roycroft J. Toxicokinetics of propylene glycol mono-t-butyl ether following intravenous or inhalation exposure in rats and mice. Inhal. Toxicol. 2004; 16: 271-90. 